\title{
Occupation times and Bessel densities
}

\author{
Yevgeniy Kovchegov*, Nick Meredith ${ }^{\dagger}$ and Eyal Nir ${ }^{\ddagger \S}$
}

\begin{abstract}
Consider a Markov process with countably many states. In order to find a one-state occupation time distribution, we use a combination of Fourier and Laplace transforms in the way that allows for the inversion of the Fourier transform. We derive a closed-form expression for the occupation time distribution in the case of a simple continuous time random walk on $\mathbb{Z}$ and represent the one state occupation density of a reversible process as a mixture of Bessel densities.
\end{abstract}

Primary Subjects: 60J35, 47N30, 47N20, 33C90, 33C10

Keywords: occupation time, Bessel functions, FRET spectroscopy, spectral measure

\section{Introduction}

The occupation time distributions for continuous-time Markov processes that live on countable state space was a subject of intense research in the $60 \mathrm{~s}, 70 \mathrm{~s}$ and early $80 \mathrm{~s}$. We would like to refer the reader to [5], [6], [13], [17], [19] and [3] for some of the results in the field. With the exception of [3], the main instrument was the multidimensional Laplace transform. More recently, a general expression in infinite sums of the occupation distributions for finite-state Markov processes was produced in [18] and [2] using essentially randomization and order statistics. The occupation times for one-dimensional nearestneighbor processes were studied by Karlin and McGregor with orthogonal polynomials in [12] following the paper of Darling and Kac [4] . Both papers considered occupation times for Markov processes when $t$ is taken to $\infty$, while the present paper concerns with closed-form expressions for a given fixed time interval $[0, t]$.

The questions addressed in this paper originate in the research done by E.Nir et al [16] in the field of single molecule Fluorescence Resonance Energy Transfer (FRET) spectroscopy, where a single molecule fluctuates between multiple states, and the experimental observable depends on a state's occupation time distribution. While working on [16] the authors have noticed that the single state occupation time densities, when computed

*Department of Mathematics, Oregon State University, Corvallis, OR 97331-4605, USA

${ }^{\dagger}$ University of California, Berkeley, CA 94720, USA

${ }^{\ddagger}$ Department of Chemistry, Ben-Gurion University, Beer-Sheva, Israel

$\S$ E.N. is supported by the Human Frontier Science Program (HFSP) 
via randomization technique (i.e. multiple infinite sums) can often be represented via modified Bessel functions of the kind

$$
I_{\rho}(z)=\sum_{k=0}^{\infty} \frac{1}{k ! \Gamma(k+\rho+1)}\left(\frac{z}{2}\right)^{2 k+\rho} \quad \text { for } \rho>-1
$$

It was observed in Section II.7 of Feller [8] that for a continuous-time random walk on $\mathbb{Z}$, the first passage time $T_{r}$ is distributed according to the Bessel density function $v_{r}(t)=e^{-t} \frac{r}{t} I_{r}(t)$, where $t>0, r \in \mathbb{Z}$ is a state, and $I_{-r}=I_{r}$. As it was the case with the first passage times, the Bessel density functions for occupation times of a two state Markov process can be obtained as a Poisson randomization of Gamma densities. In this paper, we use spectral theory in an attempt to find an analytic explanation for the relationship between occupation times and Bessel density functions. We will show a connection between a spectral measure of a generator and a Laplace transform of a single state occupation time distribution taken with respect to a time variable $t$.

\section{Approach and results}

In this section we will state the results that relate occupation times to the spectral measure of a generator, we will present a closed-form solution for a one-dimensional symmetric random walk in Theorem 2.2, and discuss the connection between occupation time distributions and modified Bessel functions.

\subsection{Spectral representation and occupation times}

Consider an irreducible continuous-time Markov process with generator $Q$ over the discrete countable state space $\Omega=\{0,1, \ldots\}$. Let $\left\{\lambda_{i, j}\right\}_{i, j \in \Omega}$ denote the rates. For a given time interval $[0, t]$, the random variable representing the time spent at the site 0 is called an occupation time at 0 . Let $f_{k}(t, x)$ denote the probability density for the occupation time at state 0 if the continuous-time process commences at state $k$. We will use the standard notation for the basis vectors $e_{0}=(1,0, \ldots)^{T}$ and $e_{j}=(0, \ldots, 0,1,0, \ldots)^{T}$.

Our first result characterizes the single-state occupation time density $f_{0}(t, x)$ as follows.

Theorem 2.1. The Laplace transform of $f_{0}(t, x)$ w.r.t. time variable $t$ can be written as

$$
E_{f_{0}}(s, x)=\frac{1}{s h(s)} \exp \left\{-\frac{x}{h(s)}\right\} \quad \text { for } s>0,
$$

where $h(s)=-\left((Q-s I)^{-1} e_{0}, e_{0}\right)$.

The domain of $h(s)$ is the resolvent set of $Q$. It was observed that if $Q$ is a bounded reversible Markov process then $-h(s)$ is a Herglotz function, i.e. it maps $\mathbb{C}_{+}$into $\mathbb{C}_{+}$. As all such functions $h$ can be represented uniquely as

$$
h(s)=-\int_{(-\infty, 0]} \frac{d \mu(x)}{x-s} \quad(\operatorname{Im}(s) \neq 0),
$$


where $\mu$ is a probability measure with compact support in $(-\infty, 0]$. See [9], [10], [11], [7], [15], and references therein.

Example: Two-state Markov processes. Consider a two-state Markov process with the generator $Q=\left(\begin{array}{cc}-\lambda & \lambda \\ \mu & -\mu\end{array}\right)$. There $(Q-s I)^{-1}=\frac{-1}{s^{2}+(\lambda+\mu) s}\left(\begin{array}{cc}\mu+s & \lambda \\ \mu & \lambda+s\end{array}\right)$ and Theorem 2.1 implies

$$
\mathrm{E}_{f_{0}}(s, x)=e^{-x(s+\lambda)} e^{\frac{\lambda \mu x}{s+\mu}}+\frac{\lambda}{s+\mu} e^{-x(s+\lambda)} e^{\frac{\lambda \mu x}{s+\mu}}
$$

Now, the equation (29.3.81) in Abramowitz and Stegun [1] contains the following Laplace transforms

$$
\int_{0}^{\infty} I_{0}(2 \sqrt{a t}) e^{-p t} d t=\frac{1}{p} e^{\frac{a}{p}} \quad \text { and } \quad \int_{0}^{\infty} \frac{1}{\sqrt{t}} I_{1}(2 \sqrt{a t}) e^{-p t} d t=\frac{1}{\sqrt{a}}\left(e^{\frac{a}{p}}-1\right),
$$

which can be rewritten as $e^{-p x} \frac{1}{p} e^{\frac{a}{p}}=\int_{x}^{\infty} I_{0}(2 \sqrt{a(t-x)}) e^{-p t} d t$ and

$$
e^{-p x} e^{\frac{a}{p}}=e^{-p x}+\sqrt{a} \int_{x}^{\infty} \frac{1}{\sqrt{t-x}} I_{1}(2 \sqrt{a(t-x)}) e^{-p t} d t
$$

Plugging in $a=\lambda \mu x$ and $p=s+\mu$, we obtain

$$
\frac{1}{s+\mu} e^{-(s+\mu) x} e^{\frac{\lambda^{2} x}{s+\mu}}=\int_{x}^{\infty} I_{0}(2 \sqrt{\lambda \mu x(t-x)}) e^{-(s+\mu) t} d t
$$

and

$$
e^{-x(s+\mu)} e^{\frac{\lambda \mu x}{s+\mu}}=e^{-(s+\mu) x}+\sqrt{\lambda \mu x} \int_{x}^{\infty} \frac{I_{1}(2 \sqrt{\lambda \mu x(t-x)})}{\sqrt{t-x}} e^{-(s+\mu) t} d t
$$

Thus

$f_{0}(t, x)=e^{-\lambda t} \delta_{t}(x)+\lambda e^{-\lambda x} e^{-\mu(t-x)} I_{0}(2 \sqrt{\lambda \mu x(t-x)})+\sqrt{\frac{\lambda \mu x}{t-x}} I_{1}(2 \sqrt{\lambda \mu x(t-x)}) e^{-\lambda x} e^{-\mu(t-x)}$ for $0 \leq x \leq t$.

The above equation was originally derived in [17] via two-dimensional Laplace transforms. One can also derive it via randomization, where the infinite sums are easily recognized to be the corresponding modified Bessel functions.

\subsection{Occupation times for simple random walks and other re- versible processes}

Surprisingly the one state occupation time density for a one-dimensional simple nearestneighbor random walk has a closed-form expression in Bessel density functions. For our 
next result, we consider

$$
Q=\left(\begin{array}{ccccc}
-r & r & 0 & 0 & \ldots \\
1 & -2 & 1 & 0 & \ldots \\
0 & 1 & -2 & 1 & \cdots \\
0 & 0 & 1 & -2 & \ddots \\
\vdots & \vdots & \vdots & \ddots & \ddots
\end{array}\right), \quad \text { where } r>0
$$

Theorem 2.2. The zero-state occupation time density for a simple nearest-neighbor random walk with the above infinitesimal generator can be expressed via modified Bessel functions as follows:

$$
\begin{gathered}
f_{0}(t, x)=e^{-r t} \delta_{t}(x)+r e^{(2-r) x-2 t} I_{0}(2 \sqrt{(t-x)(t+(r-1) x)}) \\
+\frac{r t}{\sqrt{(t-x)(t+(r-1) x)}} e^{(2-r) x-2 t} I_{1}(2 \sqrt{(t-x)(t+(r-1) x)})
\end{gathered}
$$

for $x \in[0, t]$.

Observe that $r=1$ represents the case of a simple continuous-time random walk on $\mathbb{Z}_{+}$, while the zero-state occupation time for $r=2$ corresponds to the case of a simple random walk on $\mathbb{Z}$.

Now, suppose $Q$ is a bounded reversible Markov process such as the above random walk. Then $h$ can be represented uniquely as in (1). The (spectral) probability measure $\mu$ has bounded support, say $\operatorname{supp}(\mu) \subset[-K, 0]$. Let $m_{0}, m_{1}, \ldots$ denote the moments of the spectral measure $\mu$, i.e.

$$
m_{j}=\int_{(-\infty, 0]}(-x)^{j} d \mu(x)
$$

Then, for any $z \in \mathbb{C} \backslash(-\infty, 0]$ such that $|z|>2 K$,

$$
\frac{1}{h(z)}=\frac{z}{1-m_{1} z^{-1}+m_{2} z^{-2}-\ldots}=z+m_{1}-\left(m_{2}-m_{1}^{2}\right) z^{-1}+\phi\left(z^{-1}\right) z^{-2}
$$

Recall Theorem 2.1. We will consider the inverse Laplace transform of a function $F_{0}(t, x)$, whose Laplace transform $\mathrm{E}_{F_{0}}(s, x)=\frac{1}{s} \exp \left\{-\frac{x}{h(s)}\right\}$. There $f_{0}(t, x)=-\frac{\partial}{\partial x} F_{0}(t, x)$. Take $a>2 K$ and $0 \leq x \leq t$, then the inverse Laplace transform

$$
F_{0}(t, x)=\frac{1}{2 \pi i} \int_{a-i \infty}^{a+i \infty} \frac{1}{z} \exp \left\{z(t-x)-m_{1} x+\left(m_{2}-m_{1}^{2}\right) x z^{-1}-x \phi\left(z^{-1}\right) z^{-2}\right\} d z
$$

Expanding $e^{-x \phi\left(z^{-1}\right) z^{-2}}=1+\sum_{k=2}^{\infty} v_{k}(x) z^{-k}$, we obtain

$$
\begin{aligned}
F_{0}(t, x) & =e^{-m_{1} x} I_{0}\left(2 \sqrt{\left(m_{2}-m_{1}^{2}\right)(t-x) x}\right) \\
& +e^{-m_{1} x} \sum_{k=2}^{\infty} v_{k}(x)\left(\frac{t-x}{\left(m_{2}-m_{1}^{2}\right) x}\right)^{\frac{k}{2}} I_{k}\left(2 \sqrt{\left(m_{2}-m_{1}^{2}\right)(t-x) x}\right)
\end{aligned}
$$


by (29.3.81) in Abramowitz and Stegun [1]. However, even in the case of a simple random walk, it is not obvious that the above expansion in Bessel functions using the moments of $\mu$ can be simplified to that of Theorem 2.2. In general, the following question is still to be fully explored: what properties of $\mu$ would allow for a closed-form expression of the occupation time via Bessel density functions? It seems possible to exploit the connection between spectral probability measure $\mu$ and the occupation time densities for a wider class of time reversible stochastic processes. Another interesting direction is to learn more about reinforced processes (see [14] and references therein) as they can be interpreted as occupation time driven processes.

Next we would like to discuss the limit behavior of $f_{0}(t, x)$ as $t \rightarrow \infty$. Given $t>0$, let $Z(t)$ denote the occupation time variable governed by the distribution $f_{0}(t, x)$ as derived in Theorem 2.2. As we will see in the proof of the theorem (equation (4)),

$$
h(s)=\frac{2}{(2-r) s+r \sqrt{s^{2}+4 s}}=s^{-\alpha} L\left(\frac{1}{s}\right),
$$

where $\alpha=1 / 2$ and $L(1 / s)$ is a "slowly varying" function (see [12] and [4]) that converges to $1 / r$ as $s \rightarrow 0+$. Hence, as it was the case in Karlin and McGregor [12], this $h(s)$ satisfies the main assumption for the following limit theorem in Darling and Kac [4]:

$$
\lim _{t \rightarrow \infty} P\left(\frac{Z(t)}{h(1 / t)} \leq u\right)=G_{\alpha}(u)
$$

where $G_{\alpha}(u)$ denotes the Mittag-Liffler distribution,

$$
G_{\alpha}(u)=\frac{1}{\pi \alpha} \int_{0}^{u} \sum_{n=1}^{\infty} \frac{(-1)^{n-1}}{n !} \Gamma(\alpha n+1) y^{n-1} \sin (n \pi \alpha) d y
$$

Now, since we have obtained the closed form solution in Theorem 2.2, we observe that here, the Darling-Kac limit theorem can be derived as follows. Given $u>0$,

$$
P\left(\frac{Z(t)}{h(1 / t)} \leq u\right)=\int_{0}^{u} f_{0}(t, h(1 / t) y) h(1 / t) d y
$$

Since $h(1 / t)=\frac{\sqrt{t}}{r}(1+o(1))$,

$$
f_{0}(t, h(1 / t) y) h(1 / t)=\sqrt{t} e^{(2-r) h(1 / t) y-2 t}\left[I_{0}(2 t \varphi)(1+o(1))+I_{1}(2 t \varphi)(1+o(1))\right],
$$

where

$\varphi=\sqrt{(1-h(1 / t) y / t)(1+(r-1) h(1 / t) y / t)}=1-\frac{2-r}{2} h(1 / t) y / t-\frac{r^{2}}{8}[h(1 / t)]^{2} y^{2} / t^{2}+o\left(t^{-1}\right)$

We use $I_{\nu}(z)=\frac{e^{z}}{\sqrt{2 \pi z}}(1+o(1))$ as in the equation 9.7.1 of [1] to obtain the limit

$$
\lim _{t \rightarrow \infty} P\left(\frac{Z(t)}{h(1 / t)} \leq u\right)=\frac{1}{\sqrt{\pi}} \int_{0}^{u} e^{-\frac{y^{2}}{4}} d y=G_{1 / 2}(u)
$$

via the dominated convergence theorem. 


\section{Proofs}

Proof. (Theorem 2.1) Observe that the integral equations relating $\left\{f_{k}(t, x)\right\}_{k=0,1, \ldots}$ can be produced via conditioning as follows:

$$
\begin{aligned}
f_{0}(t, x)= & e^{-\left(\sum_{m: m \neq 1} \lambda_{0, m}\right) t} \delta_{t}(x)+\sum_{k: k \neq 0} \int_{0}^{t} f_{k}(t-y, x-y) \lambda_{0, k} e^{-\left(\sum_{m: m \neq 0} \lambda_{0, m}\right) y} d y, \\
f_{j}(t, x)= & e^{-\left(\sum_{m: m \neq j} \lambda_{j, m}\right) t} \delta_{0}(x)+\sum_{k: k \neq j} \int_{0}^{t} f_{k}(t-y, x) \lambda_{j, k} e^{-\left(\sum_{m: m \neq j} \lambda_{j, m}\right) y} d y \\
& \quad \text { for } j=1,2, \ldots
\end{aligned}
$$

We plug in $\psi=t-y$ into the above equations, take the Fourier transform with respect to $x$, and simplify to get

$$
\begin{aligned}
& e^{\left(\sum_{m: m \neq 0} \lambda_{0, m}-i s_{2}\right) t} \hat{f}_{0}\left(t, s_{2}\right)=1+\sum_{k: k \neq 0} \int_{0}^{t} \hat{f}_{k}\left(\psi, s_{2}\right) \lambda_{0, k} e^{\left(\sum_{m: m \neq 0} \lambda_{0, m}-i s_{2}\right) \psi} d \psi, \\
& e^{\left(\sum_{m: m \neq j} \lambda_{j, m}\right) t} \hat{f}_{j}\left(t, s_{2}\right)=1+\sum_{k: k \neq j} \int_{0}^{t} \hat{f}_{k}\left(\psi, s_{2}\right) \lambda_{j, k} e^{\left(\sum_{m: m \neq j} \lambda_{j, m}\right) \psi} d \psi \\
& \text { for } j=1,2, \ldots \text {. }
\end{aligned}
$$

We differentiate w.r.t. variable $t$, and obtain

$$
\begin{aligned}
\left(\sum_{m: m \neq 0} \lambda_{0, m}-i s_{2}\right) \hat{f}_{0}\left(t, s_{2}\right)+\frac{\partial}{\partial t} \hat{f}_{0}\left(t, s_{2}\right) & =\sum_{k: k \neq 0} \lambda_{0, k} \hat{f}_{k}\left(t, s_{2}\right) \\
\left(\sum_{m: m \neq j} \lambda_{j, m}\right) \hat{f}_{j}\left(t, s_{2}\right)+\frac{\partial}{\partial t} \hat{f}_{j}\left(t, s_{2}\right) & =\sum_{k: k \neq j} \lambda_{j, k} \hat{f}_{k}\left(t, s_{2}\right) \quad(j=1,2, \ldots)
\end{aligned}
$$

We observe that $\hat{f}_{j}\left(0, s_{2}\right)=1$ for all $j$. Our next step is to take the Laplace transform w.r.t. variable $t$ :

$$
\begin{aligned}
\left(\sum_{m: m \neq 0} \lambda_{0, m}+s_{1}-i s_{2}\right) \mathrm{E}_{\hat{f}_{0}}\left(s_{1}, s_{2}\right) & =1+\sum_{k: k \neq 0} \lambda_{0, k} \mathrm{E}_{\hat{f}_{k}}\left(s_{1}, s_{2}\right), \\
\left(\sum_{m: m \neq j} \lambda_{j, m}+s_{1}\right) \mathrm{E}_{\hat{f}_{j}}\left(s_{1}, s_{2}\right) & =1+\sum_{k: k \neq j} \lambda_{j, k} \mathrm{E}_{\hat{f}_{k}}\left(s_{1}, s_{2}\right) \quad(j=1,2, \ldots)
\end{aligned}
$$

The above system of equations can be rewritten via the spectral decomposition of the generator operator $Q$ as follows. Let $\mathrm{E}_{\hat{f}}\left(s_{1}, s_{2}\right)=\left[\begin{array}{c}\mathrm{E}_{\hat{f}_{0}}\left(s_{1}, s_{2}\right) \\ \mathrm{E}_{\hat{f}_{1}}\left(s_{1}, s_{2}\right) \\ \vdots\end{array}\right]$ and $\mathbf{1}=\left[\begin{array}{c}1 \\ 1 \\ \vdots\end{array}\right]$. We 
proved the following spectral identity

$$
\left(Q-s_{1} I\right) \mathrm{E}_{\hat{f}}\left(s_{1}, s_{2}\right)=-\mathbf{1}-i s_{2}\left[\begin{array}{c}
\mathrm{E}_{\hat{f}_{0}}\left(s_{1}, s_{2}\right) \\
0 \\
0 \\
\vdots
\end{array}\right]
$$

Thus $\mathrm{E}_{\hat{f}}\left(s_{1}, s_{2}\right)=-\left(Q-s_{1} I\right)^{-1} \mathbf{1}-i s_{2} \mathrm{E}_{\hat{f}_{0}}\left(s_{1}, s_{2}\right)\left(Q-s_{1} I\right)^{-1} e_{0}$ and

$$
\mathrm{E}_{\hat{f}_{0}}\left(s_{1}, s_{2}\right)=-\left(\left(Q-s_{1} I\right)^{-1} \mathbf{1}, e_{0}\right)-i s_{2} \mathrm{E}_{\hat{f}_{0}}\left(s_{1}, s_{2}\right)\left(\left(Q-s_{1} I\right)^{-1} e_{0}, e_{0}\right)
$$

Therefore the Laplace-Fourier transform of $f_{0}$ can be represented as

$$
\mathrm{E}_{\hat{f}_{0}}\left(s_{1}, s_{2}\right)=\frac{-\left(\left(Q-s_{1} I\right)^{-1} \mathbf{1}, e_{0}\right)}{1+i s_{2}\left(\left(Q-s_{1} I\right)^{-1} e_{0}, e_{0}\right)}
$$

Since $\frac{1}{s_{1}}\left(Q-s_{1} I\right) \mathbf{1}=\mathbf{- 1}$, the expression in (3) can be simplified to

$$
\mathrm{E}_{\hat{f}_{0}}\left(s_{1}, s_{2}\right)=\frac{1 / s_{1}}{1-i s_{2} h\left(s_{1}\right)}
$$

where $h(s)=-\left((Q-s I)^{-1} e_{0}, e_{0}\right)$. The Fourier transform can be inverted via complex integration over a lower semi-circle contour with the radius converging to infinity, thus giving us

$$
\mathrm{E}_{f_{0}}\left(s_{1}, x\right)=\frac{1}{s_{1} h\left(s_{1}\right)} \exp \left\{-\frac{x}{h\left(s_{1}\right)}\right\}
$$

Proof. (Theorem 2.2) In the case of a simple random walk, the equation (2) translates as

$$
\begin{aligned}
\mathrm{E}_{\hat{f}_{0}}\left(s_{1}, s_{2}\right) & =\frac{1}{r+s_{1}-i s_{2}}+\frac{r}{r+s_{1}-i s_{2}} \mathrm{E}_{\hat{f}_{1}}\left(s_{1}, s_{2}\right) \\
\mathrm{E}_{\hat{f}_{1}}\left(s_{1}, s_{2}\right) & =\frac{1}{2+s_{1}}+\frac{1}{2+s_{1}} \mathrm{E}_{\hat{f}_{0}}\left(s_{1}, s_{2}\right)+\frac{1}{2+s_{1}} \mathrm{E}_{\hat{f}_{2}}\left(s_{1}, s_{2}\right) \\
\mathrm{E}_{\hat{f}_{k}}\left(s_{1}, s_{2}\right) & =\frac{1}{2+s_{1}}+\frac{1}{2+s_{1}} \mathrm{E}_{\hat{f}_{k-1}}\left(s_{1}, s_{2}\right)+\frac{1}{2+s_{1}} \mathrm{E}_{\hat{f}_{k+1}}\left(s_{1}, s_{2}\right) \quad(k=1,2, \ldots)
\end{aligned}
$$

where $\mathrm{E}_{\hat{f}_{k}}\left(s_{1}, s_{2}\right)$ again denotes the Laplace transform in the first variable $t$ and the Fourier transform in the second variable $x$ of $f_{k}(t, x)$. The functions $l_{k}\left(s_{1}, s_{2}\right)=\mathrm{E}_{\hat{f}_{k}}\left(s_{1}, s_{2}\right)-\frac{1}{s_{1}}$ satisfy the following recurrence relation

$$
l_{k}\left(s_{1}, s_{2}\right)=\frac{1}{2+s_{1}} l_{k-1}\left(s_{1}, s_{2}\right)+\frac{1}{2+s_{1}} l_{k+1}\left(s_{1}, s_{2}\right) \quad(k=1,2, \ldots)
$$


Observe that $\mathrm{E}_{\hat{f}_{k}}\left(s_{1}, 0\right)=\int_{[0,+\infty)} \int_{\mathbb{R}} e^{-s_{1} t} f_{k}(t, x) d x d t=\frac{1}{s_{1}}$ and

$\mathrm{E}_{\hat{f}_{k}}\left(s_{1}, s_{2}\right)=\int_{[0,+\infty)} \int_{\mathbb{R}} e^{-s_{1} t+i s_{2} x} f_{k}(t, x) d x d t \rightarrow \int_{[0,+\infty)} \int_{\mathbb{R}} e^{-s_{1} t+i s_{2} x} \delta_{0}(x) d x d t=\frac{1}{s_{1}}$ as $k \rightarrow \infty$

That is $l_{k}\left(s_{1}, s_{2}\right) \rightarrow 0 \quad$ as $k \rightarrow \infty$ and therefore $l_{k}\left(s_{1}, s_{2}\right)=l_{0}\left(s_{1}, s_{2}\right)\left(\frac{2+s_{1}-\sqrt{s_{1}^{2}+4 s_{1}}}{2}\right)^{k}$ as $s_{1}>0$. Thus the top recurrence equation reads

$$
\mathrm{E}_{\hat{f}_{0}}\left(s_{1}, s_{2}\right)=\frac{1}{r+s_{1}-i s_{2}}+\frac{r}{r+s_{1}-i s_{2}}\left[\left(\mathrm{E}_{\hat{f}_{0}}\left(s_{1}, s_{2}\right)-\frac{1}{s_{1}}\right) \frac{2+s_{1}-\sqrt{s_{1}^{2}+4 s_{1}}}{2}+\frac{1}{s_{1}}\right]
$$

and therefore

$$
\mathrm{E}_{\hat{f}_{0}}\left(s_{1}, s_{2}\right)=\frac{i}{2 s_{1}} \cdot \frac{(2-r) s_{1}+r \sqrt{s_{1}^{2}+4 s_{1}}}{s_{2}+\frac{i}{2}\left((2-r) s_{1}+r \sqrt{s_{1}^{2}+4 s_{1}}\right)}
$$

Once again, using complex integration, we invert the Fourier transform

$$
\mathrm{E}_{f_{0}}\left(s_{1}, x\right)=\frac{(2-r) s_{1}+r \sqrt{s_{1}^{2}+4 s_{1}}}{2 s_{1}} \exp \left\{-\frac{x}{2}\left((2-r) s_{1}+r \sqrt{s_{1}^{2}+4 s_{1}}\right)\right\}
$$

Here the spectral measure will satisfy

$$
\int_{(-\infty, 0]} \frac{d \mu(x)}{x-s}=\frac{-2}{(2-r) s+r \sqrt{s^{2}+4 s}}
$$

We will now invert the Laplace transform by decomposing $\mathrm{E}_{f_{0}}\left(s_{1}, x\right)$ as follows

$$
\mathrm{E}_{f_{0}}\left(s_{1}, x\right)=\frac{2-r}{2} \mathcal{P}_{I}+\frac{r}{2} \mathcal{P}_{I I}+2 r \mathcal{P}_{I I I}
$$

where

$$
\begin{gathered}
\mathcal{P}_{I}=\exp \left\{-\frac{x}{2}(2-r) s_{1}\right\} \cdot \exp \left\{-\frac{x}{2} r \sqrt{s_{1}^{2}+4 s_{1}}\right\}, \\
\mathcal{P}_{I I}=\frac{s_{1}}{\sqrt{s_{1}^{2}+4 s_{1}}} \exp \left\{-\frac{x}{2}(2-r) s_{1}\right\} \cdot \exp \left\{-\frac{x}{2} r \sqrt{s_{1}^{2}+4 s_{1}}\right\}
\end{gathered}
$$

and

$$
\mathcal{P}_{I I I}=\frac{1}{\sqrt{s_{1}^{2}+4 s_{1}}} \exp \left\{-\frac{x}{2}(2-r) s_{1}\right\} \cdot \exp \left\{-\frac{x}{2} r \sqrt{s_{1}^{2}+4 s_{1}}\right\}
$$

We will quote a Laplace transform formula (29.3.91) in [1]:

$$
\int_{k}^{\infty} e^{-s t} e^{-\frac{1}{2} a t} I_{0}\left(\frac{1}{2} a \sqrt{t^{2}-k^{2}}\right) d t=\frac{e^{-k \sqrt{s(s+a)}}}{\sqrt{s(s+a)}}, \quad(k \geq 0)
$$


First we will find the inverse-Laplace transform of $\mathcal{P}_{I I I}$. Taking $s=s_{1}, a=4$ and $k=\frac{r x}{2}$ in (29.3.91) of [1], we get

$$
\int_{\frac{r x}{2}}^{\infty} e^{-s_{1} t} e^{-2 t} I_{0}\left(2 \sqrt{t^{2}-\left(\frac{r x}{2}\right)^{2}}\right) d t=\frac{e^{-\frac{r x}{2} \sqrt{s_{1}\left(s_{1}+4\right)}}}{\sqrt{s_{1}\left(s_{1}+4\right)}}
$$

Multiplying both sides of the above equation by $\exp \left\{-\frac{(2-r) x}{2} s_{1}\right\}$, and changing the variable to $t:=t+\frac{(2-r) x}{2}$, obtain

$$
\mathcal{P}_{I I I}=\int_{x}^{\infty} e^{-s_{1} t} e^{(2-r) x-2 t} I_{0}(2 \sqrt{(t-x)(t+(r-1) x)}) d t
$$

Therefore, the inverse of $\mathcal{P}_{I I I}$ is

$$
\mathcal{L}^{-1}\left(\mathcal{P}_{I I I}\right)=e^{(2-r) x-2 t} I_{0}(2 \sqrt{(t-x)(t+(r-1) x)}) \cdot \mathbf{1}_{\{x \leq t\}}
$$

We differentiate $\frac{\partial}{\partial t}$ and integrate by parts in (5):

$$
\mathcal{L}\left(\frac{\partial}{\partial t}\left[e^{(2-r) x-2 t} I_{0}(2 \sqrt{(t-x)(t+(r-1) x)}) \cdot \mathbf{1}_{\{x \leq t\}}\right]\right)=\mathcal{P}_{I I}-e^{-r x} e^{-s_{1} x}
$$

Hence

$$
\begin{gathered}
\mathcal{L}^{-1}\left(\mathcal{P}_{I I}\right)=e^{-r t} \delta_{0}(t-x)-2 e^{(2-r) x-2 t} I_{0}(2 \sqrt{(t-x)(t+(r-1) x)}) \cdot \mathbf{1}_{\{x \leq t\}} \\
+\frac{2 t-(2-r) x}{\sqrt{(t-x)(t+(r-1) x)}} e^{(2-r) x-2 t} I_{1}(2 \sqrt{(t-x)(t+(r-1) x)}) \cdot \mathbf{1}_{\{x \leq t\}}
\end{gathered}
$$

In order for us to invert $\mathcal{P}_{I}$, we will need (29.3.96) of [1], that states the following

$$
\int_{k}^{\infty} e^{-s t} \frac{a k}{\sqrt{t^{2}-k^{2}}} I_{1}\left(a \sqrt{t^{2}-k^{2}}\right) d t=e^{-k \sqrt{s^{2}-a^{2}}}-e^{-k s}, \quad(k>0)
$$

Here we let $s=s_{1}+2, a=2$ and $k=\frac{r x}{2}$, thus obtaining

$$
\mathcal{P}_{I}=e^{-r x} e^{-s_{1} x}+\int_{\frac{r x}{2}}^{\infty} e^{-s_{1}\left(t+\frac{(2-r) x}{2}\right)} \frac{r x e^{-2 t}}{\sqrt{t^{2}-\left(\frac{r x}{2}\right)^{2}}} I_{1}\left(2 \sqrt{t^{2}-\left(\frac{r x}{2}\right)^{2}}\right) d t
$$

Once again changing the variable to $t:=t+\frac{(2-r) x}{2}$, get

$$
\mathcal{P}_{I}=e^{-r x} e^{-s_{1} x}+\int_{x}^{\infty} e^{-s_{1} t} \frac{r x e^{(2-r) x-2 t}}{\sqrt{(t-x)(t+(r-1) x)}} I_{1}(2 \sqrt{(t-x)(t+(r-1) x)}) d t
$$

and

$$
\mathcal{L}^{-1}\left(\mathcal{P}_{I}\right)=e^{-r t} \delta_{0}(t-x)+\frac{r x e^{(2-r) x-2 t}}{\sqrt{(t-x)(t+(r-1) x)}} I_{1}(2 \sqrt{(t-x)(t+(r-1) x)}) \cdot \mathbf{1}_{\{x \leq t\}}
$$

We add up all three terms together, thus proving the theorem. 


\section{Acknowledgment}

The authors wish to thank R.Burton and M.Ossiander for sharing thoughts on the subject of this paper. We are grateful to the anonymous referee for suggesting that we include a discussion on limit behavior of $f_{0}(t, x)$ following Theorem 2.2.

\section{References}

[1] M.Abramowitz and I.A. Stegun (Eds.), Handbook of mathematical functions with formulas, graphs, and mathematical tables U.S. Department of Commerce (1972)

[2] M.Bladt, B.Meini, M.F.Neuts and B.Sericola, Distributions of reward functions on continuous time Markov chains in Matrix-analytic methods: theory and application (Eds. G. Latouche and P. Taylor) (2002), pp.39-62

[3] L.Bondesson, On occupation times for quasi-Markov processes J.Appl.Prob., 18, (1981), pp.297-301

[4] D.A.Darling and M.Kac, On occupation times for Markoff processes Transactions of AMS, 84, (1957), pp.444-458

[5] J.N.Darroch, Identities for passage times with applications to recurrent events and homogeneous differential functions J.Appl.Prob., 3, (1966), pp.435-444

[6] J.N.Darroch and K.Morris, Passage-time generating functions for continuous-time finite Markov chains J.Appl.Prob., 5, (1968), pp.414-426

[7] P.Deift, Orthogonal Polynomials and Random Matrices: A Riemann-Hilbert Approach Amer. Math. Soc., Providance, RI, (2000)

[8] W.Feller, An Introduction to Probability Theory and Its Applications (Volume II) Second Edition, John Wiley \& Sons (1971)

[9] M.L.Gorbachuk and V.I.Gorbachuk, M.G.Krein's Lectures on Entire Operators Birkhäuser Verlag (1997)

[10] S.Karlin and J.L.McGregor, The differential equations of birth and death processes and the Stieltjes problem Transactions of AMS, 85, (1957), pp.489-546

[11] S.Karlin and J.L.McGregor, The classification of birth and death processes Transactions of AMS, 86, (1957), pp.366-400

[12] S.Karlin and J.L.McGregor, Occupation time laws for birth and death processes Proc. 4th Berkeley Symp. Math. Statist. Prob., 2, (1962), pp.249-272

[13] L.M.Kovaleva, On the occupation time in a given state for the simples semi-Markov system Teor. Veroyat. i Mat. Stat., 1, (1970), pp.100-107

[14] Y.Kovchegov, Multi-particle processes with reinforcements Journal of Theoretical Probability, 21, (2008), pp.437-448

[15] Y.Kovchegov, Orthogonality and probability: beyond nearest neighbor transitions Electronic Communications in Probability, 14 (2009), pp.90-103 
[16] E.Nir, X.Michalet, K.Hamadani, T.A.Laurence, D.Neuhauser, Y.Kovchegov and S.Weiss, Shot-noise limited single-molecule FRET histogram: comparison between theory and experiments Journal of Physical Chemistry B, Vol.110, No.44 (2006), pp.22103-22124

[17] P.J.Pedler, Occupation times for two-state Markov chains J.Appl.Prob., 8, (1971), pp.381-390

[18] B.Sericola, Occupation times in Markov processes Stochastic Models, 16, (2000), pp.479-510

[19] W.S.Hsia, The joint probability density function of the occupation time of a three-state problem J.Appl.Prob., 13, (1971), pp.57-64 